Research Article

\title{
Guided Wave Energy Transfer in Composite Sandwich Structures and Application to Defect Detection
}

\author{
Siavash Shoja (D), Viktor Berbyuk (D), and Anders Boström \\ Department of Mechanics and Maritime Sciences, Chalmers University of Technology, 41296 Gothenburg, Sweden \\ Correspondence should be addressed to Siavash Shoja; siavash.shoja@chalmers.se
}

Received 28 May 2018; Revised 29 August 2018; Accepted 17 September 2018; Published 1 November 2018

Academic Editor: Antonella Cecchi

Copyright (C) 2018 Siavash Shoja et al. This is an open access article distributed under the Creative Commons Attribution License, which permits unrestricted use, distribution, and reproduction in any medium, provided the original work is properly cited.

In this study, energy transmission of the guided waves propagating in composite sandwich structures is investigated in a wide range of frequencies using numerical simulations. The effects of different potential defects on the guided wave energy transmission are explored in such structures. Furthermore, the accuracy of homogenization methods for finite element modelling of guided wave propagation in sandwich structures is studied with the aim of reducing the computational burden of the simulations in the low range of frequencies. A 2D finite element model is developed and verified by comparing the results with the dispersion curves. In order to examine homogenization methods, the homogenized stiffness matrices of the sandwich material and the laminate skin are calculated using classical laminate theory. Results show that core-skin debonding causes absence of wave energy leakage from the skin to the core material in that region in a specific range of frequencies. The results are also obtained for the delamination within the skin and compared with the healthy material. Finally, for the guided waves in the low range of frequencies, it is possible to use the homogenization methods to create the finite element models and reduce the solution time.

\section{Introduction}

Composite sandwich structures are types of materials which have a lightweight but thick core attached between two thin but stiffer skins, often made of composite laminates. These types of materials are extensively used in various applications due to their special characteristics such as high bending stiffness, high strength, and excellent dynamic properties together with low weight $[1,2]$.

One of the major challenges in using composite sandwich structures is that they are more likely to be subjected to debonding and failure. This is due to their large weak interfaces between adjacent materials with very different stiffness and strength properties [3]. An increase in the size of the debonded region in composite sandwich materials can lead to fatal failure in larger structures. Therefore, early detection of the debonding using some nondestructive testing (NDT) techniques can prevent catastrophic failures and reduces the cost of maintenance. Due to the special characteristics of the guided wave- (GW-) based inspection techniques, they have been used for inspection of sandwich structures.

An early study on the application of GW propagation to defect detection in composite sandwich structures was carried out by Bertoni and Park [4] in which the GW is introduced as a potential tool for NDT of sandwich structures. In their study, an aluminium plate is bonded with a semi-infinite honeycomb panel and the formal solution for the GW is calculated. Their calculation shows the leakage of wave energy from the skin to the honeycomb. Also, they realized in case of a sandwich plate, a honeycomb core, and two skin plates, the wave energy radiated by the leaky waves couples with the lower plate. Furthermore, the majority of the studies show that, in a specific range of frequencies, debonding between the laminate and the core material causes an increase in the signal compared to the baseline. This is the range of frequencies in which Lamb waves propagating in the top laminate skin shows leaky behaviour meaning that it attenuates quickly because of loss of energy to the core material. These studies are based on both 
numerical and experimental investigation, and attenuation characteristics of the core material are accounted for [5-10].

It is also shown that, at frequencies lower than in the abovementioned studies, debonding between the core material and the skin of the sandwich structure causes creation of reflected waves $[11,12]$. Information regarding the severity of a defect can be obtained by processing the reflected and transmitted waves in the time or frequency domains [13-16].

Further studies on the effects of defects on GW propagation in sandwich structures can be performed using numerical simulations. The numerical models have been mainly based on the finite element (FE) method, and commercial codes are usually used to simulate the propagation of the GW. Moreover, by transient simulation of GW propagation, which can be done using the FE models, it is possible to calculate the amplitude and consequently energy transmission of the propagated waves. The models are either $2 \mathrm{D}$ with plane strain approximation $[5,17-19]$, or $3 \mathrm{D}$ models using shell or solid elements $[6,8,10,13,20,21]$. In the $3 \mathrm{D}$ FE models, the sandwich structures are modelled using two plates with shell elements for the top and bottom layers representing the laminate skin with a core which is modelled by solid elements $[6,13,22]$. Modelling the foam or honeycomb core layer using solid elements requires homogenization assumptions. Studies show that assuming a homogeneous isotropic material for the foam or honeycomb core leads to reasonable accuracy for the fundamental wave modes in the low range of frequencies [10, 23]. The simulations are mostly performed using explicit dynamic procedures, and Abaqus is the commercial code which has been widely used for these types of studies $[5,6,8,13,20]$.

Most of the abovementioned studies shows different behaviours of GW in different frequency ranges when they interact with the defect. This brings an emphasis on the need of studying GW propagation problem in a wide range of frequencies to observe the phenomena. Since amplification of the signal in a defected structure comparing to the healthy one is previously reported $([5,12,24])$, the focus should be on the change of the amplitude during the propagation and interaction with defects. This can be done by taking into account the energy transmission of GW as a criterion.

FE modelling of any sandwich structure using the mentioned methods requires considering special convergence criteria which are functions of the excitation frequency $[25,26]$. This makes the FE models computationally heavy and in cases of large structures almost impossible to solve. Therefore, the size of the models should be reduced in terms of the number of elements. Homogenizing the composite material into a single orthotropic layer reduces the number of elements in the models.

In the current work, a study is performed based on a $2 \mathrm{D}$ FE model. The model is first verified by comparing the results in the frequency-wavenumber domain with the dispersion curves. Effects of debonding between the laminate skin and the core material on GW are studied by further computations in the range of frequency-thickness product from $110 \mathrm{kHz}-\mathrm{mm}$ to $1100 \mathrm{kHz}-\mathrm{mm}$. The results are compared with the results of a similar model in which a delamination is placed between the plies of the top laminate instead of a debonding between the laminate and core material. Moreover, classical laminate theory (CLT) is used to homogenize the sandwich composite structure, and the possibility of using such models for GW propagation in the very low range of frequencies is investigated. In the following debonding between the core material and the laminate skin is referred to as "debonding" and delamination in the laminate skin is referred to as "delamination."

\section{Numerical Model}

In this section, the FE model of wave propagation in a composite sandwich plate is presented. ABAQUS with explicit dynamic procedure is used to create the model and perform simulations with the assumptions of linear elasticity. Mass, stiffness of the material, and external force are defined in the model. The assumed material is a composite sandwich plate with length $1.5 \mathrm{~m}$ and total thickness $7.4 \mathrm{~mm}$. The sandwich plate consists of two laminate skins on top and bottom of an isotropic light core. The laminate skins have four plies with the stacking sequence of $[0 / 90]_{2}$ and $0.3 \mathrm{~mm}$ ply thickness. Table 1 shows the properties of the lamina and the core material.

The geometry of the model is a $2 \mathrm{D}$ rectangle, and it is discretized using 2D elements with a plane strain assumption (CPE4R). The rectangle is divided into nine layers in the thickness direction, of which the top four represent the top laminate, the middle one is the isotropic core material, and the bottom four are the bottom laminate. The fibre orientation angle is defined by switching the longitudinal and transverse ( $x$ and $y$ ) properties of the $90^{\circ}$ plies given in Table 1. Using this method, it is possible to include 0 and $90^{\circ}$ fibre orientation angles to the proper plies. However, to include other fibre orientation angles, a 3D model is needed.

To avoid the reflections from the ends of the geometry disturbing the incident wave, so-called absorbing regions are introduced at the two ends of the geometry. Absorbing layers using the increasing damping (ALID) method is used to damp the waves arriving to the regions. In this method, the domain is extended with layers of the same material but increasing damping factor [27]. The Rayleigh damping model is used according to

$$
C=\alpha M+\beta K,
$$

where $C, M$, and $K$ are, respectively, damping, mass, and stiffness matrices. $\alpha$ and $\beta$ are the constants of proportionality. For the absorbing regions, $\alpha$ is exponentially increased from a small number to $2 \times 10^{5} \mathrm{~s}^{-1}$. This exponential increase of the damping factor avoids the unwanted reflection between the elements of the ALID regions. The plain strain assumption together with the ALID regions makes the FE model to be as close as possible to a model of a plate with infinite size. In order to avoid attenuation of GW during propagation and study the energy transmission, damping is only applied in the absorbing regions and not in the main part of the geometry.

The excitation force is applied as a nodal force in the $z$ direction to a node located on the top layer of the plate. This 
TABLE 1: Mechanical properties of the lamina and the core material.

\begin{tabular}{|c|c|c|c|c|c|c|c|c|c|c|c|c|}
\hline \multicolumn{10}{|c|}{ Lamina } & \multicolumn{3}{|c|}{ Core } \\
\hline$E_{x}$ & $E_{y}$ & $E_{z}$ & $\begin{array}{c}G_{x y} \\
(\mathrm{GPa})\end{array}$ & $G_{x z}$ & $G_{y z}$ & $v_{x y}$ & $v_{x z}$ & $v_{y z}$ & $\begin{array}{c}\rho \\
\left(\mathrm{kg} / \mathrm{m}^{3}\right)\end{array}$ & $\begin{array}{c}E \\
(\mathrm{MPa}) \\
\end{array}$ & $v$ & $\begin{array}{c}\rho \\
\left(\mathrm{kg} / \mathrm{m}^{3}\right)\end{array}$ \\
\hline 130 & 7 & 7 & 4 & 4 & 2.5 & 0.3 & 0.3 & 0.4 & 1500 & 61.4 & 0.3 & 100 \\
\hline
\end{tabular}

type of excitation force creates both symmetric and asymmetric wave modes in the structure and might make it difficult to understand the behaviour of GW in the material. However, since this type of one sided excitation is common in the majority of applications, it is used in this work to study the response of the material. To verify the model and create symmetric and asymmetric wave modes separately, the material can be excited at two nodes on two opposite sides of the plate. The force is applied as a tone-burst signal with the centre frequency varying from $15 \mathrm{kHz}$ to $150 \mathrm{kHz}$.

It has been shown that, in explicit FE modelling of GW propagation, temporal and spatial resolution must be small enough for the results to converge [28]. The criteria for the largest time step $(\Delta t)$ and element size $\left(l_{\mathrm{e}}\right)$ are, respectively,

$$
\begin{aligned}
& \Delta t=\frac{1}{10 f_{\text {max }}}, \\
& l_{\mathrm{e}}=\frac{\lambda_{\text {min }}}{10},
\end{aligned}
$$

where $\lambda_{\min }$ is the smallest wavelength and $f_{\max }$ is the highest frequency of excitation.

Figure 1 shows a schematic view of the FE model. The response of the system is observed by extracting the nodal acceleration in the $z$-direction at 100 nodes equally distributed between the points $0.01 \mathrm{~m}$ to $1.01 \mathrm{~m}$ from the excitation point. These measurement nodes are located on the surface of the top and bottom laminates and give the spatial sampling frequency $630 \mathrm{rad} / \mathrm{m}$. Every run of the simulation lasts for $1.5 \mathrm{~ms}$ which is enough for the A0 mode to propagate once through the plate and reach its far end.

Debonding between the laminate and the isotropic core material and delamination between the plies of the laminates have been modelled using the extended finite element method (XFEM) implemented in Abaqus. In this method, the presence of discontinuities is modelled by special local enriched functions in conjunction with additional degrees of freedom $[29,30]$. Since the displacement due to the GW propagation is negligible compared to the typical gap size between the debonded layers in a sandwich plate, no interaction is specified between the two debonded surfaces. The same procedure is applied to model a delamination between the plies of the skin laminate.

2.1. Homogenized Model. Assuming that the bond between the plies of the laminate is perfect, it is possible to homogenize the material into a single layer with equivalent characteristics. The CLT, which is derived from classical plate theory, calculates stiffness properties of the laminate by integration of in-plane stresses in the direction normal to the laminates surface [31]. Since homogenization using CLT makes a major reduction in FE model size, it has been widely used in FE calculations of composite laminates.

Assuming $[\mathbf{Q}]$ to be the stiffness matrix of an individual lamina that is calculated using the mechanical properties presented in Table 1, it is possible to include the orientation angles by multiplying by the transformation matrices:

$$
\left[\mathbf{Q}_{\mathrm{c}}\right]=\left[\mathbf{T}_{1}\right]^{-1}[\mathbf{Q}]\left[\mathbf{T}_{2}\right] \text {, }
$$

where $\left[\mathbf{T}_{1}\right]$ and $\left[\mathbf{T}_{2}\right]$ are, respectively, stress and strain transformation matrices. Here, $\left[\mathbf{Q}_{c}\right]$ is the stiffness matrix of a ply respecting its fibre direction. The extensional stiffness matrix can be calculated by integrating over the ply thickness and summing:

$$
[\mathbf{A}]=\sum_{j=1}^{N} \int_{h_{j}}^{h_{j+1}}\left[\mathbf{Q}_{\mathbf{c}}\right]_{j} \mathrm{~d} z,
$$

where $N$ is the total number of plies, $h_{j}$ is the thickness of ply $j$, and $z$ is the coordinate of the ply in the thickness direction. The explicit description of CLT is shown in [31].

To calculate the equivalent stiffness matrix, the extensional stiffness matrix is normalized by the thickness:

$$
[\mathbf{K}]=\frac{[\mathbf{A}]}{t_{\text {tot }}},
$$

where $t_{\text {tot }}$ is the total plate thickness. For the out-of-plane stiffness terms $\left(K_{44}, K_{45}, K_{54}, K_{55}\right)$, the common shear correction factor of 5/6 is applied in the calculation [31].

To examine if it is possible to homogenize the whole sandwich material using the homogenization method, the isotropic core should be considered as one of the plies. Moreover, a general rule of mixtures is used to calculate the equivalent density of the sandwich plate. The calculated stiffness and density are applied on the same 2D model for which the homogenized material characteristics are used. A homogenized 2D model of the top laminate skin is developed to compare the results with the two other models. The calculated material properties of the homogenized sandwich plate and the homogenized laminate skin are presented in Table 2.

2.2. Model Verification. To verify the FE model, the response of the system should be studied for a wide range of frequencies. One way to perform the verification is to calculate the dispersion curves for the same material and compare them with the results obtain from the simulations. The dispersion curves are calculated using the method based on the Floquet-Bloch theory [32]. In this method, the eigenfrequencies are calculated for a unit cell for which the Floquet periodic boundary conditions are applied for the boundaries in the wave propagation direction. Next, the 


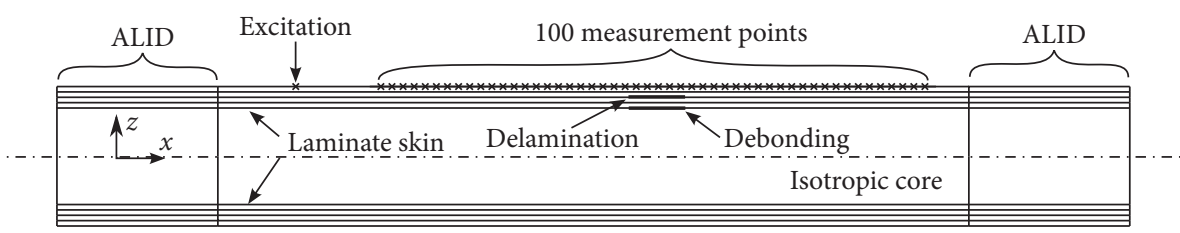

FIGURE 1: Schematic view of the numerical model including the approximate locations of the defects.

TABLE 2: Equivalent stiffness and density of the homogenized plates.

\begin{tabular}{lcccccccccc}
\hline & & \multicolumn{4}{c}{ Stiffness matrix $(\mathrm{GPa})$} & \multicolumn{4}{c}{ Density $\left(\mathrm{kg} / \mathrm{m}^{3}\right)$} \\
& $K_{11}$ & $K_{12}$ & $K_{13}$ & $K_{22}$ & $K_{23}$ & $K_{33}$ & $K_{44}$ & $K_{55}$ & $K_{66}$ & $\rho$ \\
\hline Plate & 24.5 & 13.9 & 13.9 & 24.5 & 2.52 & 7.02 & 0.89 & 0.89 & 1.3 & 554 \\
Skin & 75.2 & 42.86 & 42.86 & 75.2 & 2.5 & 7 & 2.71 & 2.71 & 4 & 1500 \\
\hline
\end{tabular}

dispersion curves can be calculated using the eigenfrequencies and wavenumbers.

The results obtained from the FE simulation are converted from the time-space domain into the frequencywavenumber domain using the $2 \mathrm{D}$ Fourier transform. By this conversion, it is possible to depict the area of the dispersion curves as high magnitude regions. This method has been previously introduced by Alleyne and Cawley [33] and is mostly used to obtain the dispersion curves using FE simulations or experimental measurements.

To obtain the results for a wide range of frequencies, a sine pulse is used as the excitation signal. The signal is applied as forces on two nodes on the top and bottom of the sandwich material, once in the same direction, then in the the opposite directions (Figure 2). Using this method, it is possible to generate only symmetric or asymmetric wave modes in every simulation. The results are summed together and compared with the dispersion curves obtained previously, shown in Figure 3.

The analysis shows that there is fair agreement between the results obtained from the FE model and the dispersion curves, for frequencies lower than $40 \mathrm{kHz}$. For the higher wave modes, the disagreement between the results increases by increasing the frequency which these modes are not in focus of the current study.

\section{Results and Discussion}

3.1. GW Energy Transfer. The dispersion behaviour of the A0 and S0 wave modes presented in Figure 3 shows a drop in phase velocity of the S0 wave mode at a frequency of approximately $25 \mathrm{kHz}$. This drop has as a consequence that both wave modes have approximately equal velocities above this frequency. By exciting the plate with a single force on one side of the plate, the displacement due to A0 and S0 wave modes cancels each other on the other side of the plate. Therefore, pure symmetric and asymmetric wave modes are not visible in the results above this frequency. This can be seen in Figure 4 where the wave pattern is shown at the excitation frequency $75 \mathrm{kHz}$. It is possible to see that considerably higher magnitudes are created in the top laminate skin in the beginning. By increasing the propagation time, the high wave magnitudes are transferring to the core material and then reache the bottom skin.

To better understand the phenomenon, the wave energy is calculated and followed in the top and bottom skins along the length of the sandwich material. The normalized transmitted energy is calculated as

$$
E_{\text {trans }}=\int_{t_{\text {start }}}^{t_{\text {end }}} a^{2}(t) \mathrm{d} t,
$$

where $a(t)$ is the normalized acceleration [23]. The A0 and S0 wave modes are the dominant ones in the propagating wave; therefore, the calculated energy corresponds mostly to the energy of these two wave modes at one measurement node. By repeating the same calculations for all the measurement nodes along the length of the sandwich material, it is possible to obtain the transformation of wave energy between the top and bottom laminates. Figure 5 shows the results obtained for different excitation frequencies between $15 \mathrm{kHz}$ and $150 \mathrm{kHz}$.

The results show that, at $15 \mathrm{kHz}$, the difference between the transmitted energy in the top and bottom laminates is almost negligible, meaning that the A0 and S0 are created in the whole thickness with different phase velocities so that they do not interact with each other. By increasing the frequency, it is possible to see that the wave energy starts transmitting back and forth between the top and bottom laminates. This is the frequency range where the A0 and S0 wave modes have very nearly the same phase velocities (Figure 3). Moreover, it is possible to see that the number of intervals where the wave energy transmits between the top and bottom laminates changes by changing the difference between the phase velocities of these two wave modes. By reducing the difference between the velocities of the two wave modes, the number of energy transmissions reduces. This phenomenon is best visible at the frequency of $150 \mathrm{kHz}$ at which the amount of transmitted energy between the top and bottom laminates is reduced. Here, one can predict that when the wavelength is smaller than the thickness of the top laminate, the wave propagates on the surface of the laminate and it does not reach into the core material. 


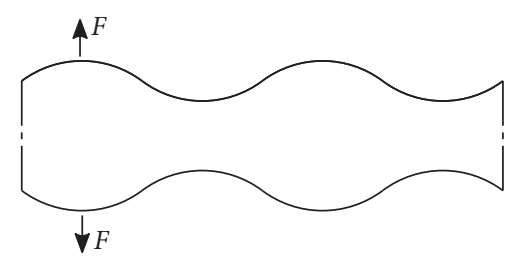

(a)

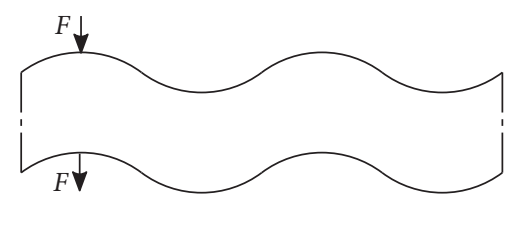

(b)

Figure 2: Creation of symmetric (a) and asymmetric (b) wave modes in a transient FE simulation.

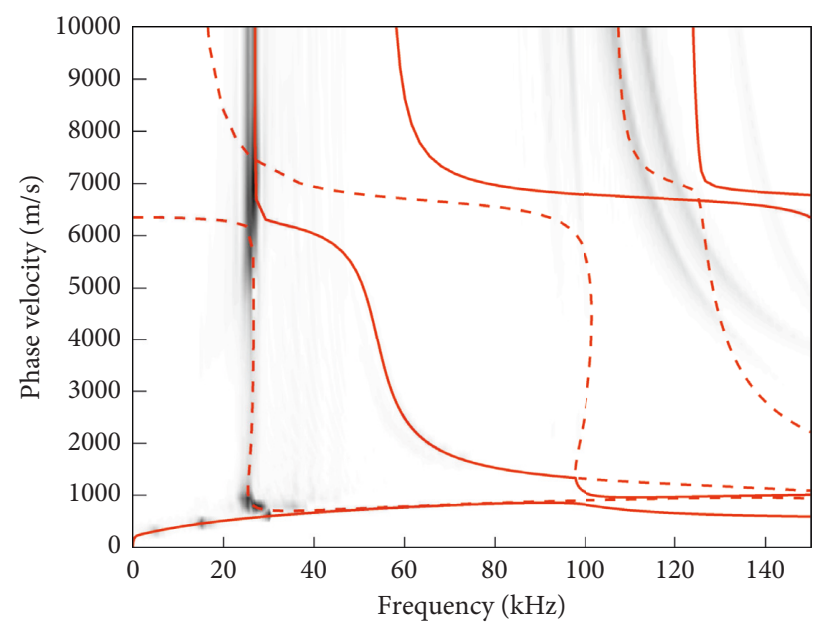

FIGURE 3: A comparison of the dispersion curves of the sandwich plate and the model's response in the frequency-wavenumber domain (the solid red lines are the asymmetric modes, and the dotted red lines are the symmetric modes).
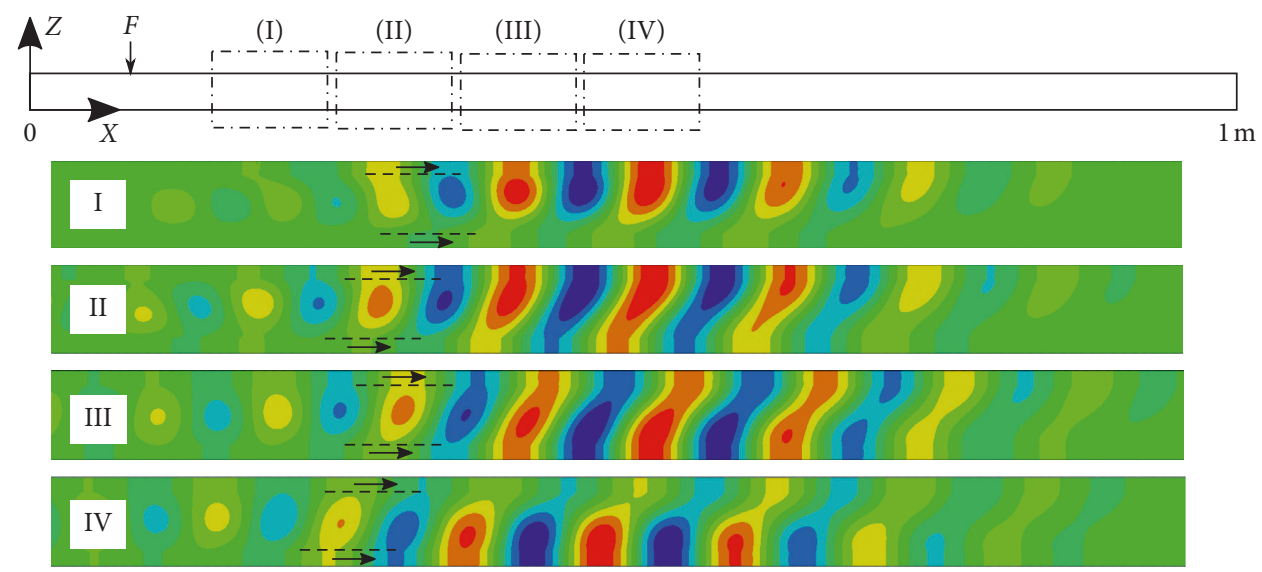

Figure 4: Wave pattern at the excitation frequency $75 \mathrm{kHz}$ in different regions of the plate for (I) $t=0.09 \mathrm{~ms}$, (II) $t=0.15 \mathrm{~ms}$, (III) $t=0.21 \mathrm{~ms}$, and (IV) $t=0.27 \mathrm{~ms}$. The dotted lines show the approximate location of the laminate skins, and the arrows show the direction of propagation.

3.2. Interaction of $G W$ and Debonding. Figure 5 shows the energy transmission in the top and bottom laminate skins in a range of frequencies between $15 \mathrm{kHz}$ and $150 \mathrm{kHz}$. It is observed that debonding causes fluctuation in the energy of the transmitted wave in both the top and bottom skins at $15 \mathrm{kHz}$ (Figure 5(a)).

The effects of debonding on GW propagation are, however, different at higher frequencies. Since the debonding is located between the core material and the top skins, the energy stops transmitting into the core material from the layer and the magnitude of the energy remains constant. At the same time, this causes a drop in the transmitted energy to the bottom layer. This can be seen by following the red curves in Figure 5 for all the frequencies higher than $15 \mathrm{kHz}$. These are the range of frequencies where the $\mathrm{A} 0$ and $\mathrm{S} 0$ wave modes have approximately equal phase velocities, and the GWs propagating in this range are referred to as the leaky Lamb wave in previous work $[5-8,20]$. 


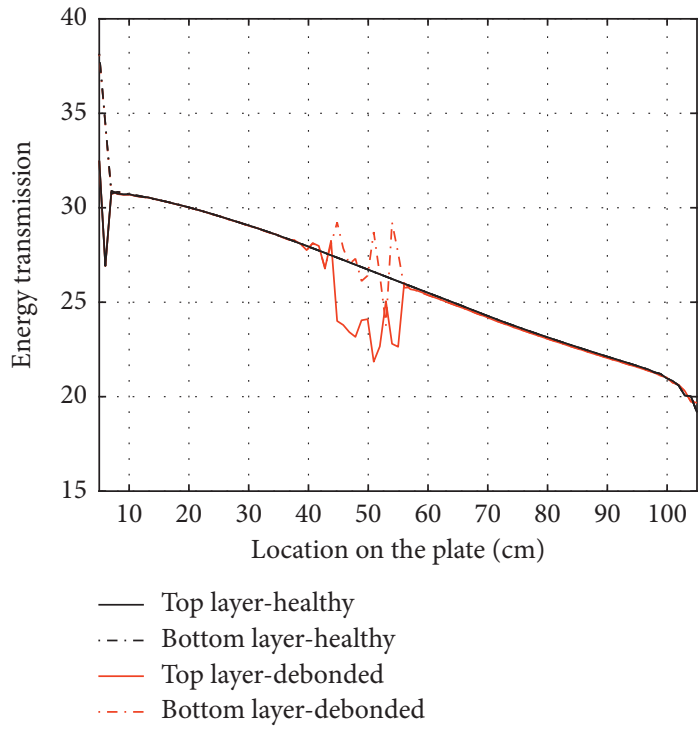

(a)

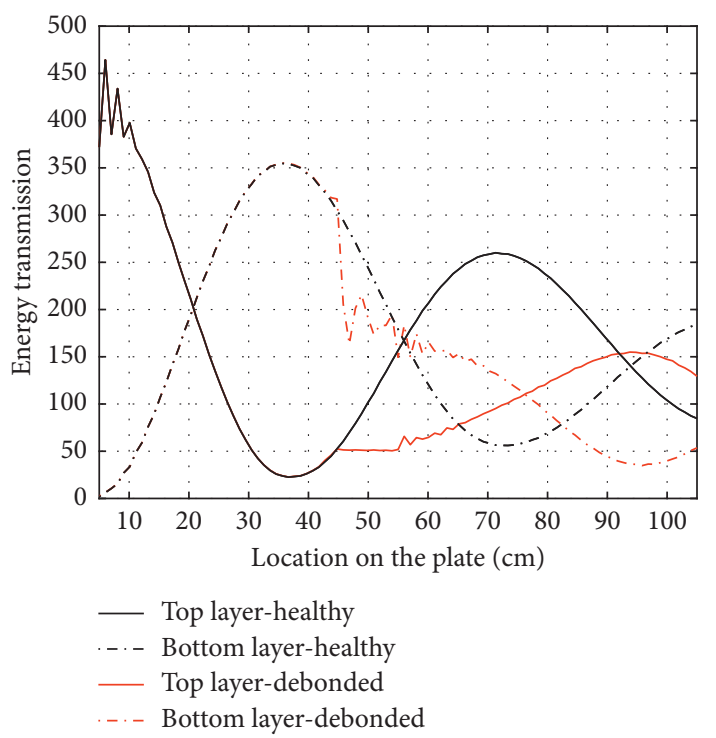

(c)

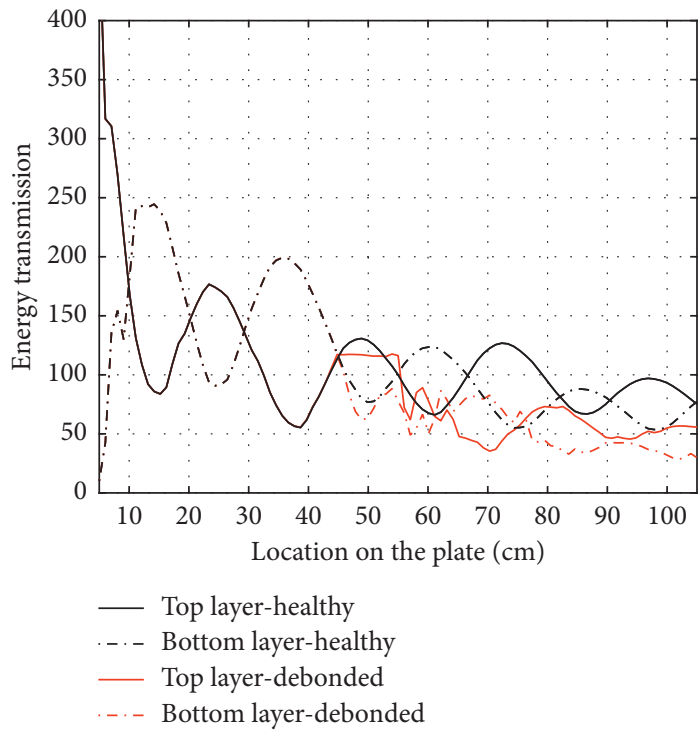

(e)

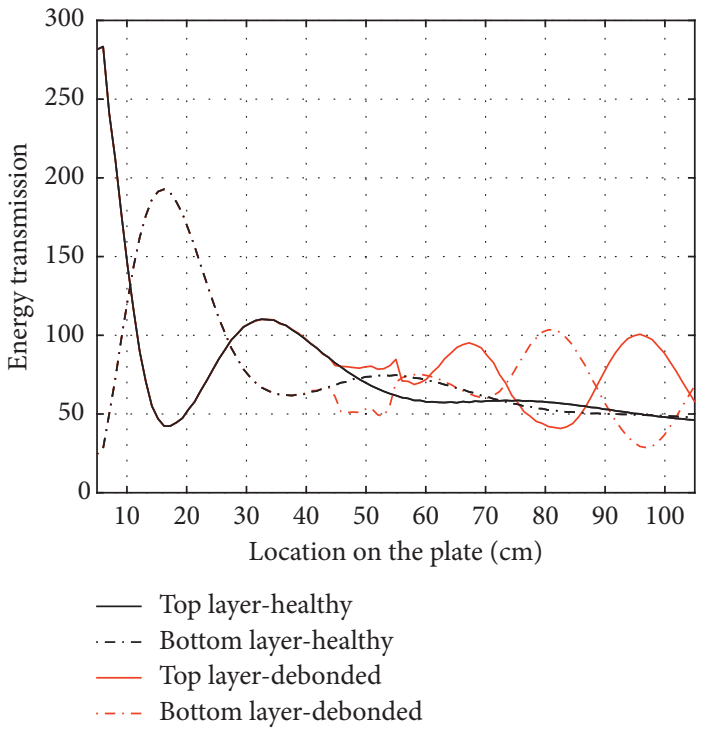

(b)

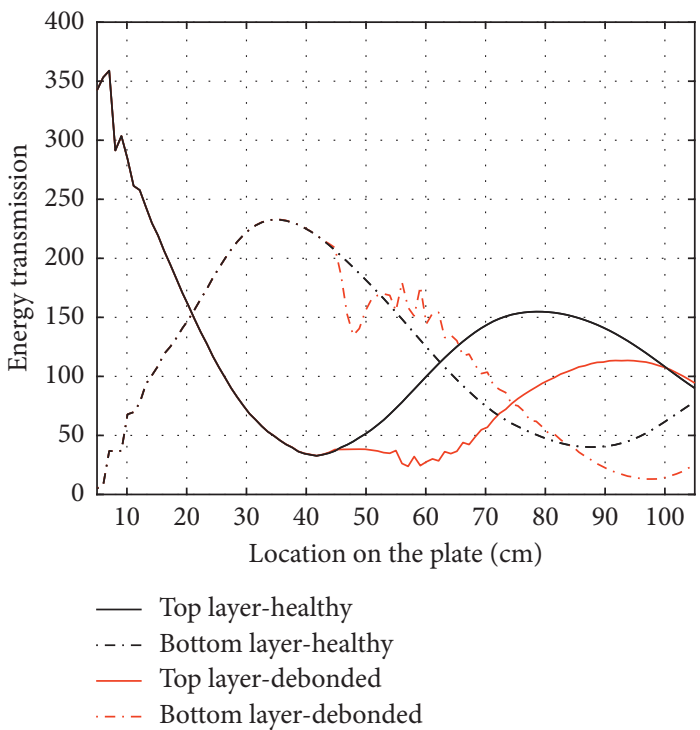

(d)

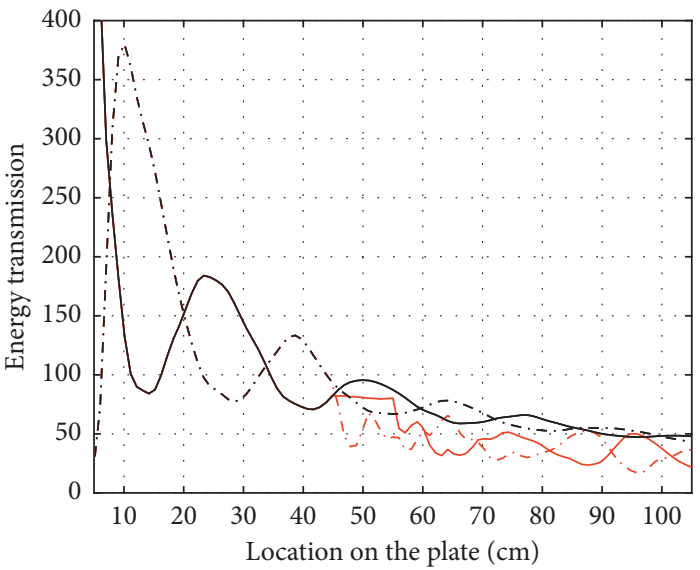

_- Top layer-healthy
-.- Bottom layer-healthy
_ Top layer-debonded
..- Bottom layer-debonded

(f)

Figure 5: Energy response in the top and bottom laminate skins for both healthy and debonded plates for excitation frequencies of (a) $15 \mathrm{kHz}$, (b) $40 \mathrm{kHz}$, (c) $60 \mathrm{kHz}$, (d) $80 \mathrm{kHz}$, (e) $100 \mathrm{kHz}$, and (f) $150 \mathrm{kHz}$. 


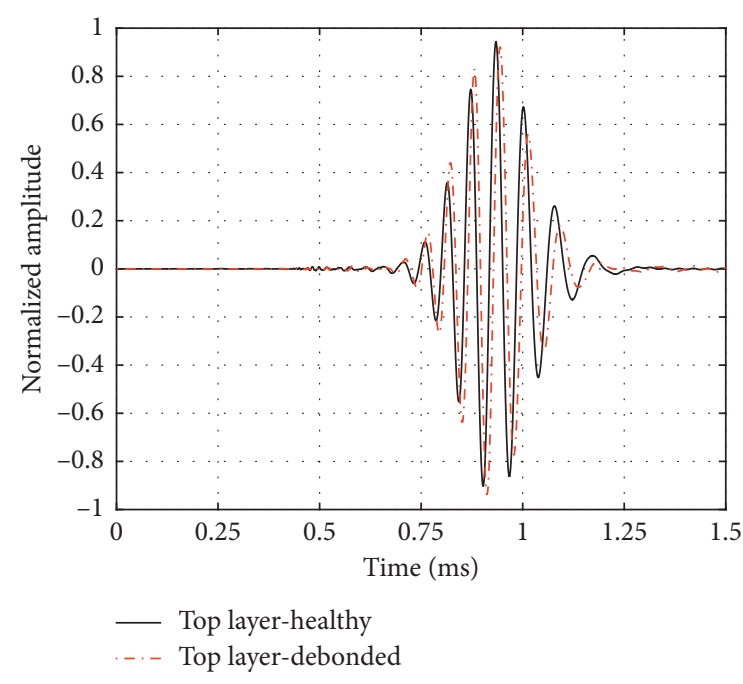

(a)

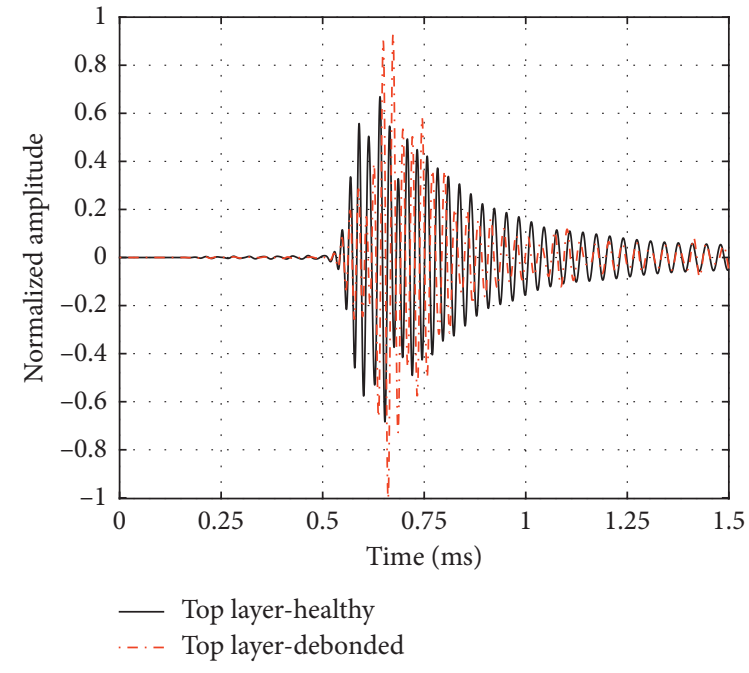

(b)

Figure 6: Response of the system in the time domain for both healthy and debonded plates for excitation frequencies of (a) $15 \mathrm{kHz}$ and (b) $40 \mathrm{kHz}$ at a measurement node after the debonded region.

Due to the clear change in energy transmission of the GW, the waves propagating in this range of frequencies are capable of detecting debonding in sandwich structures.

For a better understanding, a comparison is shown in the time domain for the two frequencies $15 \mathrm{kHz}$ and $40 \mathrm{kHz}$ in Figure 6. The results show that the interaction of GW with a debonding in the composite material delays the time signal (reduction in the wave velocity) and creates reflections. As expected, this behaviour is different at higher frequencies where the GW show "leaky" behaviour and the debonding in the composite plate cause significant increase in the amplitude of the propagating wave comparing to the healthy material. This is due to absence of energy transmission into the core material in the debonded region.

3.3. Interaction of $G W$ and Delamination. To compare the effect of delamination on GW propagation in sandwich plates with the previous results, a delamination is created in the middle of the top laminate. Similar simulations are carried out as in the previous section, and energy transmission by the GW propagation is calculated using the mentioned method. The results are shown in Figure 7 together with energy transmission in the healthy material. It is observed that the creation of a delamination in the top laminate causes fluctuations in the energy transmission, especially in the top laminate. However, no special pattern has been found similar to the previous section. This shows that the special behaviour of GW at higher frequencies does not affect the energy transmission of the propagated wave when interacting with delaminations.

This is further investigated by comparing the results in the time domain for the two different frequencies $15 \mathrm{kHz}$ and $40 \mathrm{kHz}$ in Figure 8 . The results show that the delamination delays the time signal and creates reflected waves at both frequencies.
3.4. Homogenized Model for GW Propagation. As previously mentioned, GWs are created in the low range of frequencies in sandwich plates. To observe the effect of the homogenization method in this range of frequencies, the results are obtained using the two homogenized models of sandwich plate and top skin and are compared with the results of the layerwise model. A comparison of the time signals is shown in Figure 9.

The results show that significant disagreement is observed comparing the response of the homogenized model with that of the layerwise one. Moreover, by modelling the homogenized top laminate skin and comparing the response signal with the layerwise model, it is seen that good agreement is reached. This shows that propagation of GW in the sandwich material is to some extent similar to propagation of GW in its laminate skin at the excitation frequency of $15 \mathrm{kHz}$. Here, it is possible to simplify the model by creating the homogenized skin instead of the layerwise sandwich plate. Therefore, in large 3D sandwich models, homogenized shell elements can be used instead of layerwise models with solid elements; however, each case needs to be examined individually.

\section{Conclusion}

Studying the GW energy transfer in sandwich structures shows that depending on the frequency of excitation, the effect of defects on GW propagation varies. At low excitation frequencies, both skin delamination and core-to-skin debonding cause reduction in GW velocity. However, at higher frequencies, where the "leaky" behaviour is reported, skin-to-core debonding prevents leakage of wave energy into the core material and consequently causes significant amplification of the propagating wave compared to the healthy material. This does not occur in case of a skin delamination. Therefore, debonding and delamination can be distinguished from each other in this range of frequencies. Furthermore, in this range of frequencies, due to approximately 

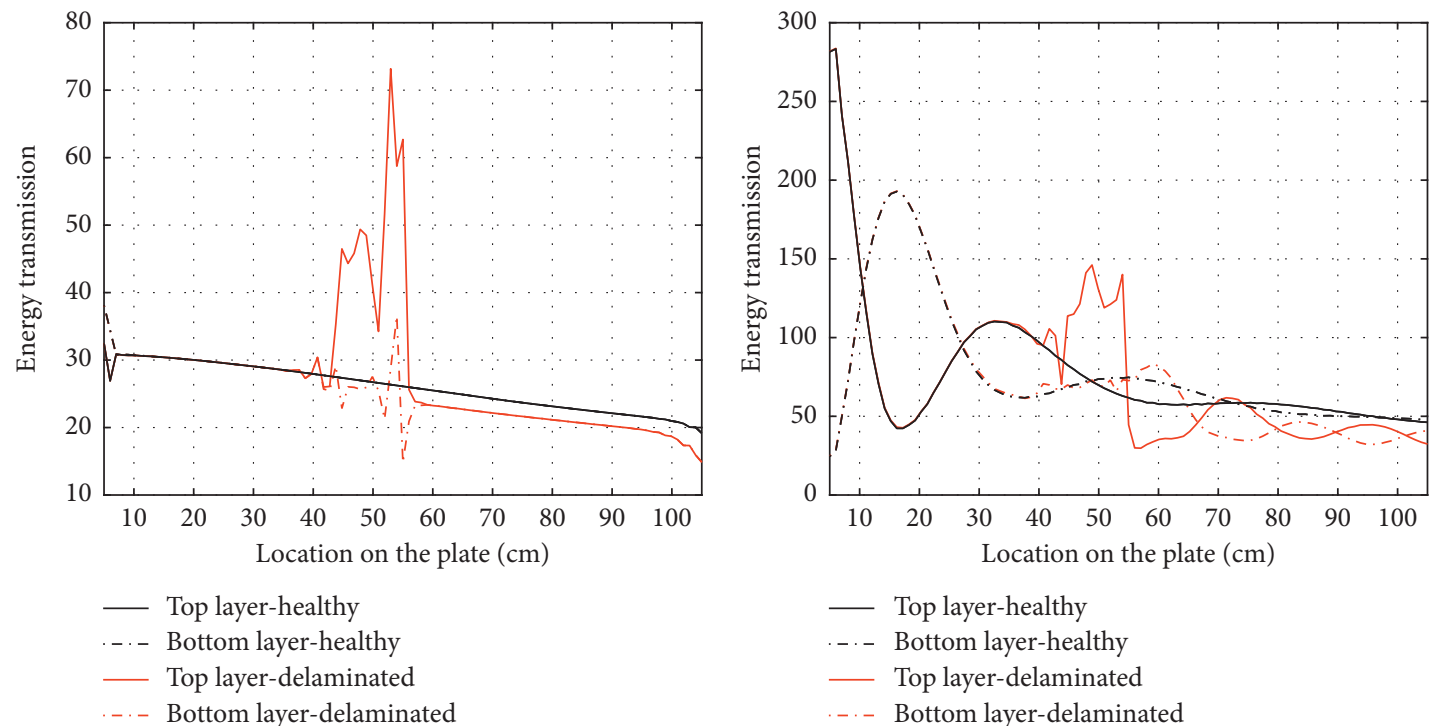

(a)

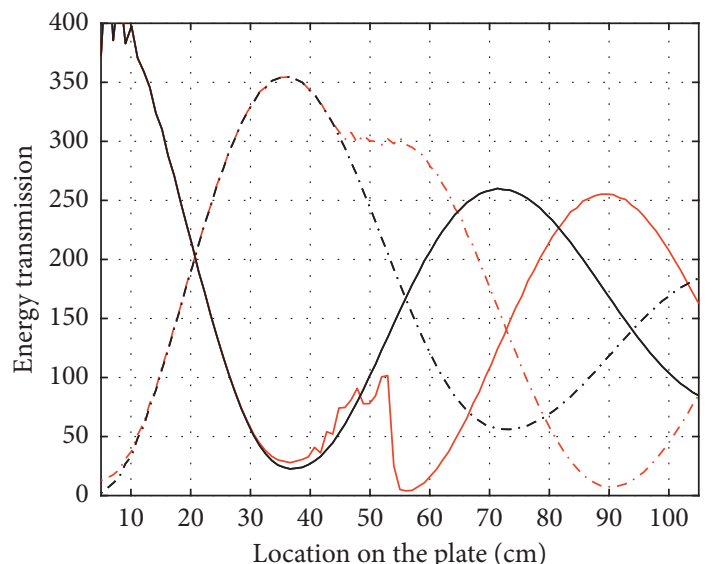

(b)

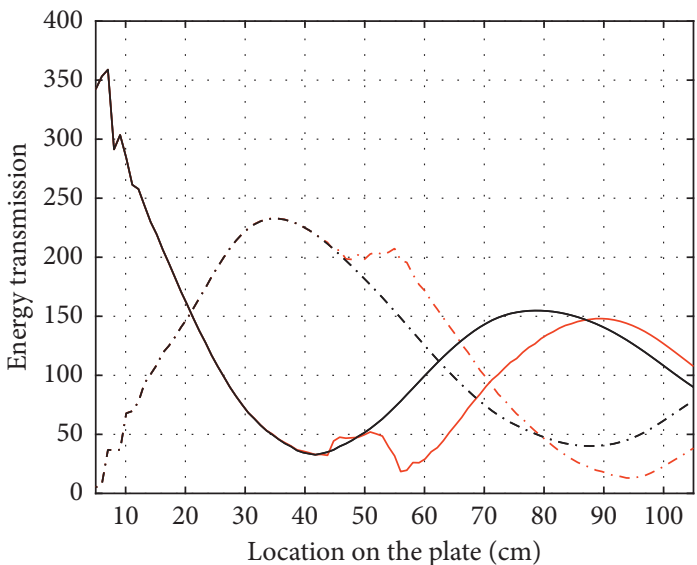

_ Top layer-healthy

-. Bottom layer-healthy

— Top layer-delaminated

-.- Bottom layer-delaminated

(c)

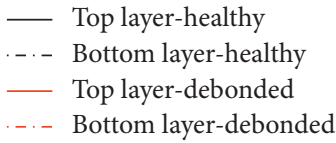

(d)
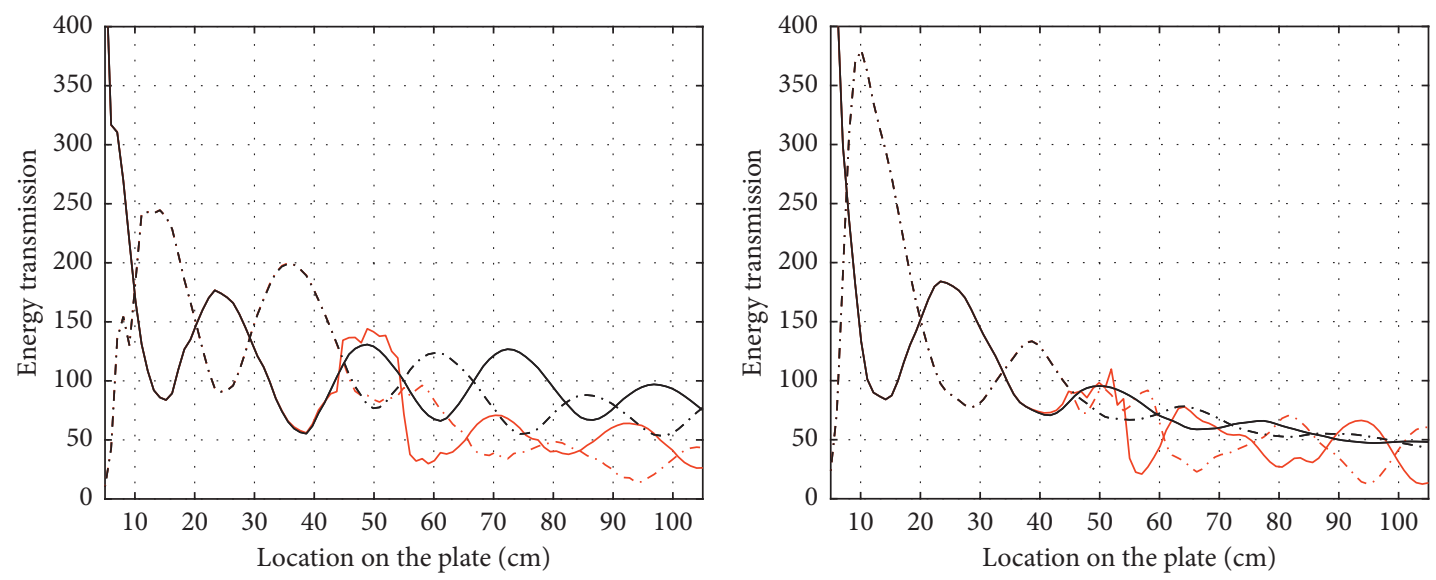

_ Top layer-healthy

-..- Bottom layer-healthy

_ Top layer-delaminated

...- Bottom layer-delaminated

(e)

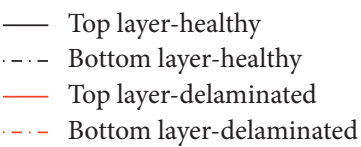

(f)

Figure 7: Energy response in the top and bottom laminate skins for both healthy and delaminated plates for excitation frequencies of (a) $15 \mathrm{kHz}$, (b) $40 \mathrm{kHz}$, (c) $60 \mathrm{kHz}$, (d) $80 \mathrm{kHz}$, (e) $100 \mathrm{kHz}$, and (f) $150 \mathrm{kHz}$. 


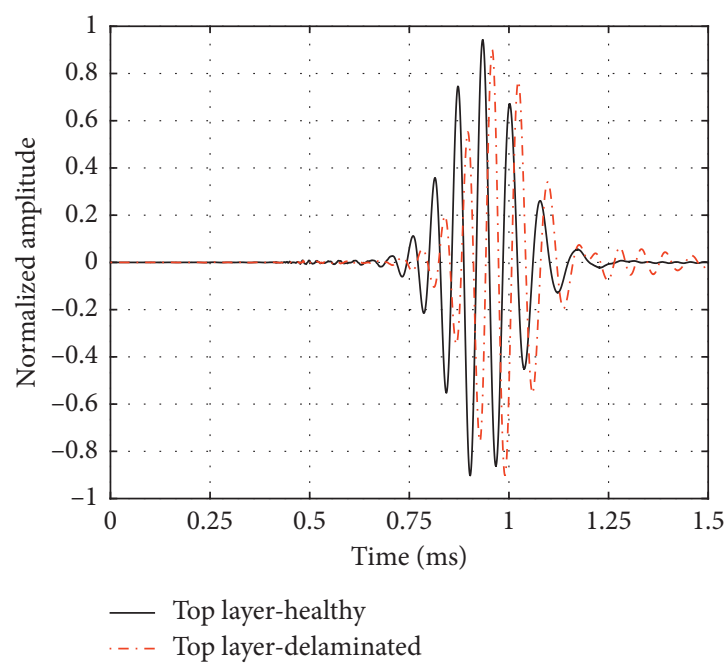

(a)

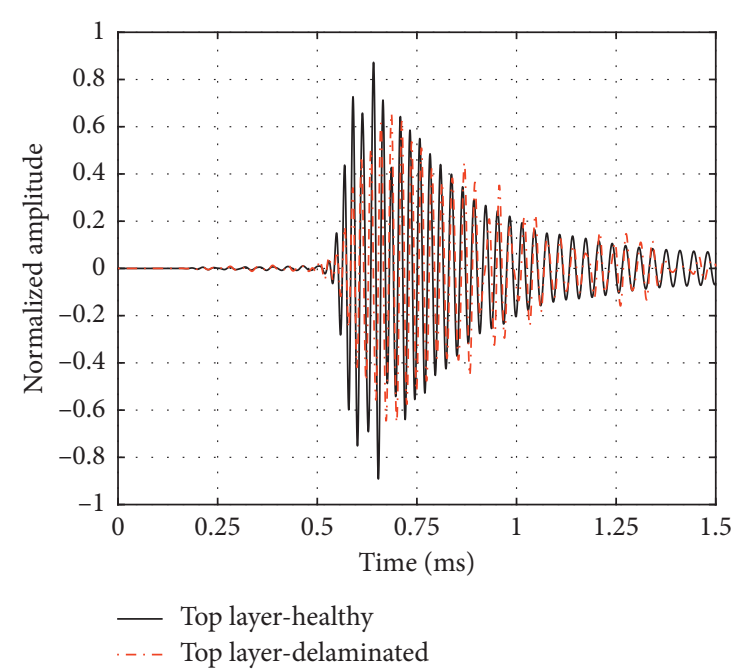

(b)

Figure 8: Response of the system in the time domain for both healthy and delaminated plates for excitation frequencies of (a) $15 \mathrm{kHz}$ and (b) $40 \mathrm{kHz}$ at a measurement node after the debonded region.

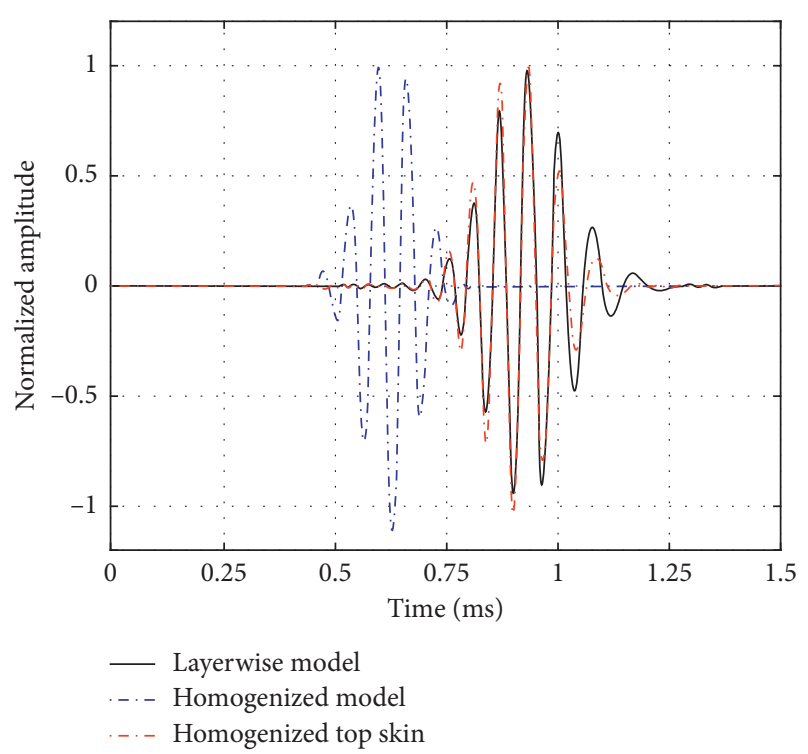

Figure 9: A comparison between the responses of the model in the time domain for layerwise, homogenized plate, and homogenized top skin for the excitation frequency $15 \mathrm{kHz}$.

similar phase velocities of A0 and S0 wave modes, a combination of both wave modes propagates in the material instead of pure symmetric and asymmetric modes.

Finally, it is shown that the FE model can be simplified by modelling the homogenized top skin instead of the sandwich plate in the low range of frequencies. This can create significant reduction in solution time which is important in FE modelling of wave propagation in large composite structures.

\section{Data Availability}

Data regarding the presented results are available through e-mail upon request.

\section{Conflicts of Interest}

The authors declare that they have no conflicts of interest.

\section{Acknowledgments}

The simulations were performed on resources at the Chalmers Centre for Computational Science and Engineering (C3SE) provided by the Swedish National Infrastructure for Computing (SNIC).

\section{References}

[1] A. Krzyhak, M. Mazur, M. Gajewski, K. Drozd, A. Komorek, and P. Przybyłek, "Sandwich structured composites for aeronautics: methods of manufacturing affecting some mechanical properties," International Journal of Aerospace Engineering, vol. 2016, Article ID 7816912, 10 pages, 2016.

[2] G. R. Gillich, Z. I. Praisach, M. Abdel Wahab, and O. Vasile, "Localization of transversal cracks in sandwich beams and evaluation of their severity," Shock and Vibration, vol. 2014, Article ID 607125, 10 pages, 2014.

[3] O. T. Thomsen, "Sandwich materials for wind turbine blades present and future," Journal of Sandwich Structures \& Materials, vol. 11, no. 1, pp. 7-26, 2009.

[4] H. L. Bertoni and S. K. Park, "Propagation of elastic waves in honeycomb panels for application to rapid inspection techniques," Journal of Nondestructive Evaluation, vol. 2, no. 3-4, pp. 209-217, 1981.

[5] S. Sikdar, S. Banerjee, and G. Ashish, "Ultrasonic guided wave propagation and disbond identification in a honeycomb composite sandwich structure using bonded piezoelectric wafer transducers," Journal of Intelligent Material Systems and Structures, vol. 27, no. 13, pp. 1767-1779, 2016.

[6] S. Mustapha and L. Ye, "Propagation behaviour of guided waves in tapered sandwich structures and debonding identification using time reversal," Wave Motion, vol. 57, pp. 154-170, 2015. 
[7] T. R. Hay, L. Wei, J. L. Rose, and T. Hayashi, "Rapid inspection of composite skin-honeycomb core structures with ultrasonic guided waves," Journal of Composite Materials, vol. 37, no. 10, pp. 929-939, 2003.

[8] S. Sikdar and S. Banerjee, "Identification of disbond and high density core region in a honeycomb composite sandwich structure using ultrasonic guided waves," Composite Structures, vol. 152, pp. 568-578, 2016.

[9] N. Bourasseau, E. Moulin, C. Delebarre, and P. Bonniau, "Radome health monitoring with lamb waves: experimental approach," NDT \& E International, vol. 33, no. 6, pp. 393-400, 2000.

[10] F. Song, G. L. Huang, and K. Hudson, "Guided wave propagation in honeycomb sandwich structures using a piezoelectric actuator/sensor system," Smart Materials and Structures, vol. 18, no. 12, article 125007, 2009.

[11] C. Soutis and K. Diamanti, "Active sensing of impact damage in composite sandwich panels by low frequency lamb waves," Aeronautical Journal, vol. 112, no. 1131, pp. 279-283, 2008.

[12] K. Diamanti, C. Soutis, and J. M. Hodgkinson, "Lamb waves for the non-destructive inspection of monolithic and sandwich composite beams," Composites Part A: Applied Science and Manufacturing, vol. 36, no. 2, pp. 189-195, 2005.

[13] S. Mustapha, L. Ye, D. Wang, and Y. Lu, "Assessment of debonding in sandwich CF/EP composite beams using A0 lamb wave at low frequency," Composite Structures, vol. 93, no. 2, pp. 483-491, 2011.

[14] S. Mustapha, L. Ye, D. Wang, and Y. Lu, "Debonding detection in composite sandwich structures based on guided waves," AIAA Journal, vol. 50, no. 8, pp. 1697-1706, 2012.

[15] S. Thwaites and N. H. Clark, "Non-destructive testing of honeycomb sandwich structures using elastic waves," Journal of Sound and Vibration, vol. 187, no. 2, pp. 253-269, 1995.

[16] J. Wu, Z. Ma, and Y. Zhang, "A time-frequency research for ultrasonic guided wave generated from the debonding based on a novel time-frequency analysis technique," Shock and Vibration, vol. 2017, Article ID 5686984, 11 pages, 2017.

[17] D. L. Osmont, D. Devillers, and F. Taillade, "Health monitoring of sandwich plates based on the analysis of the interaction of lamb waves with damages," in Proceedings of SPIE's 8th Annual International Symposium on Smart Structures and Materials, vol. 4327, p. 12, SPIE, Newport Beach, CA, USA, March 2001.

[18] M. Castaings and B. Hosten, "Guided waves propagating in sandwich structures made of anisotropic, viscoelastic, composite materials," Journal of the Acoustical Society of America, vol. 113, no. 5, pp. 2622-2634, 2003.

[19] H. Gao, S. Ali, and B. Lopez, "Efficient detection of delamination in multilayered structures using ultrasonic guided wave EMATs," NDT \& E International, vol. 43, no. 4, pp. 316-322, 2010.

[20] S. Mustapha and L. Ye, "Leaky and non-leaky behaviours of guided waves in CF/EP sandwich structures," Wave Motion, vol. 51, no. 6, pp. 905-918, 2014.

[21] S. Sikdar, P. Kudela, M. Radzieński, A. Kundu, and W. Ostachowicz, "Online detection of barely visible low-speed impact damage in 3D-core sandwich composite structure," Composite Structures, vol. 185, pp. 646-655, 2018.

[22] P. Zhang, Y. Cheng, and J. Liu, "Numerical analysis of dynamic response of corrugated core sandwich panels subjected to near-field air blast loading," Shock and Vibration, vol. 2014, Article ID 180674, 16 pages, 2014.

[23] S. M. H. Hosseini, A. Kharaghani, C. Kirsch, and U. Gabbert, "Numerical simulation of lamb wave propagation in metallic foam sandwich structures: a parametric study," Composite Structures, vol. 97, pp. 387-400, 2013.

[24] F. Song, G. Huang, and G. Hu, "Online guided wave-based debonding detection in honeycomb sandwich structures," AIAA Journal, vol. 50, no. 2, pp. 284-293, 2012.

[25] S. Shoja, V. Berbyuk, and A. Boström, "Guided wave-based approach for ice detection on wind turbine blades," Wind Engineering, vol. 42, no. 5, pp. 483-495, 2018.

[26] S. Shoja, V. Berbyuk, and A. Boström, "Delamination detection in composite laminates using low frequency guided waves: numerical simulations," Composite Structures, vol. 203, pp. 826-834, 2018.

[27] M. Drozdz, L. Moreau, M. Castaings, M. J. S. Lowe, and P. Cawley, "Efficient numerical modelling of absorbing regions for boundaries of guided waves problems," AIP Conference Proceedings, vol. 820, no. 1, pp. 126-133, 2006.

[28] F. Moser, L. J. Jacobs, and J. Qu, "Modeling elastic wave propagation in waveguides with the finite element method," NDT \& E International, vol. 32, no. 4, pp. 225-234, 1999.

[29] M. Smith, ABAQUS/Standard User's Manual, Version 6.9, Simulia, Johnston, RI, USA, 2009.

[30] J.-H. Song, P. M. A. Areias, and T. Belytschko, "A method for dynamic crack and shear band propagation with phantom nodes," International Journal for Numerical Methods in Engineering, vol. 67, no. 6, pp. 868-893, 2006.

[31] B. Agarwal, L. Broutman, and K. Chandrashekhara, Analysis and Performance of Fiber Composites, Wiley, Hoboken, NJ, USA, 2006.

[32] P. G. García and J. P. Fernández-Álvarez, "Floquet-bloch theory and its application to the dispersion curves of nonperiodic layered systems," Mathematical Problems in Engineering, vol. 2015, Article ID 475364, 12 pages, 2015.

[33] D. Alleyne and P. Cawley, "A two-dimensional fourier transform method for the measurement of propagating multimode signals," Journal of the Acoustical Society of America, vol. 89, no. 3, pp. 1159-1168, 1991. 


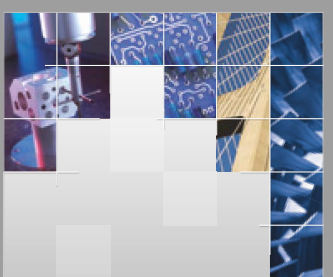

\section{Enfincering}
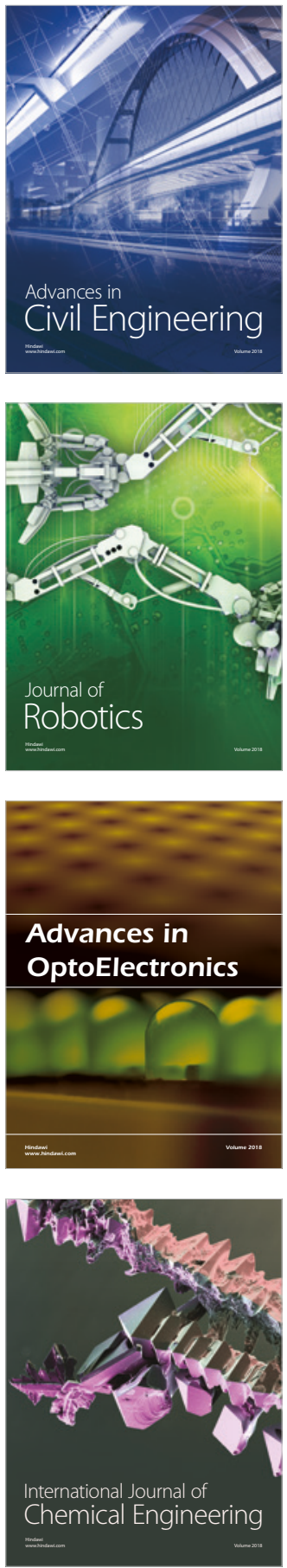

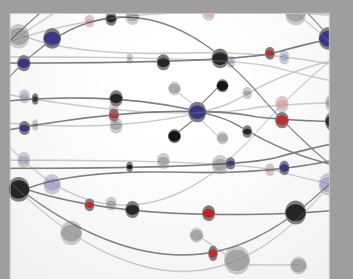

\section{Rotating \\ Machinery}

The Scientific World Journal

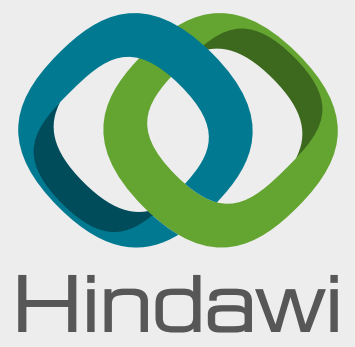

Submit your manuscripts at

www.hindawi.com
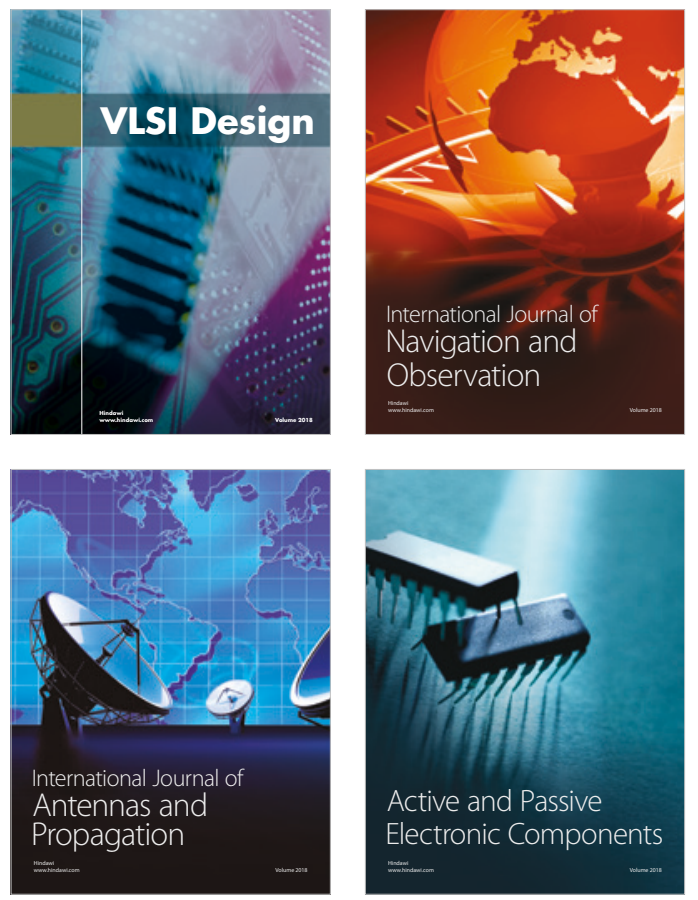
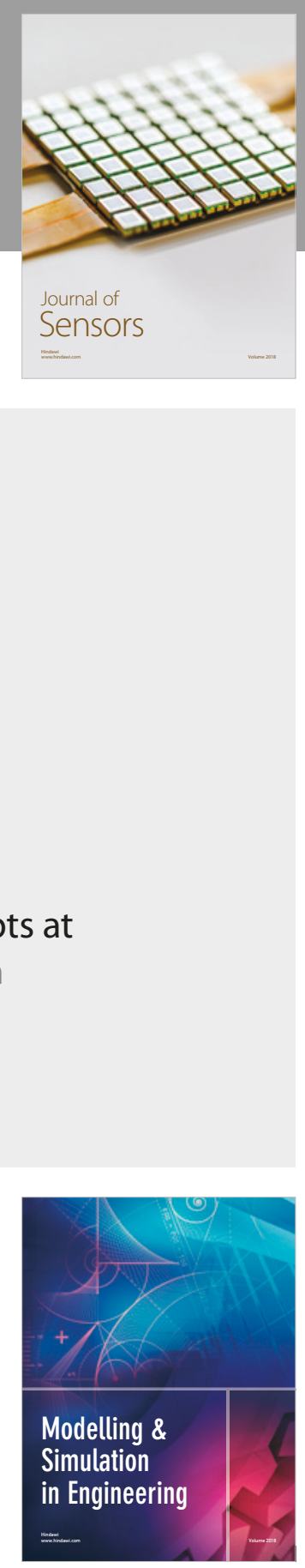

\section{Advances \\ Multimedia}
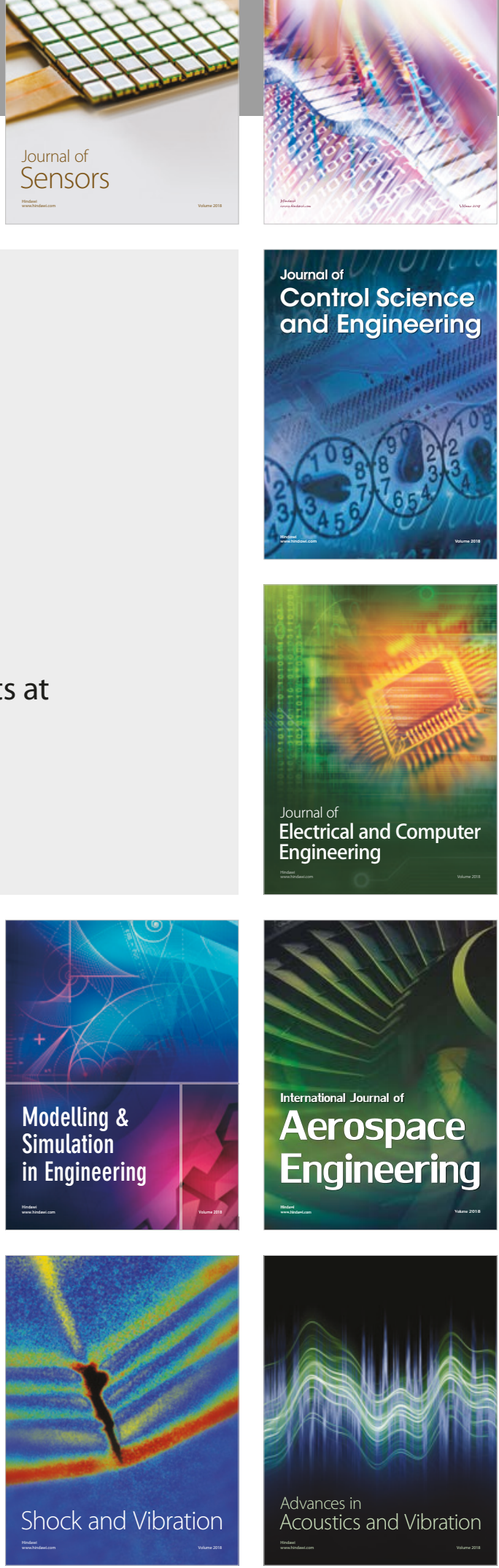